\title{
Population Data on Finland 1900-1998 ${ }^{1}$
}

\section{UPDATED BY VEERA REUNA}

Resident population on 31 December

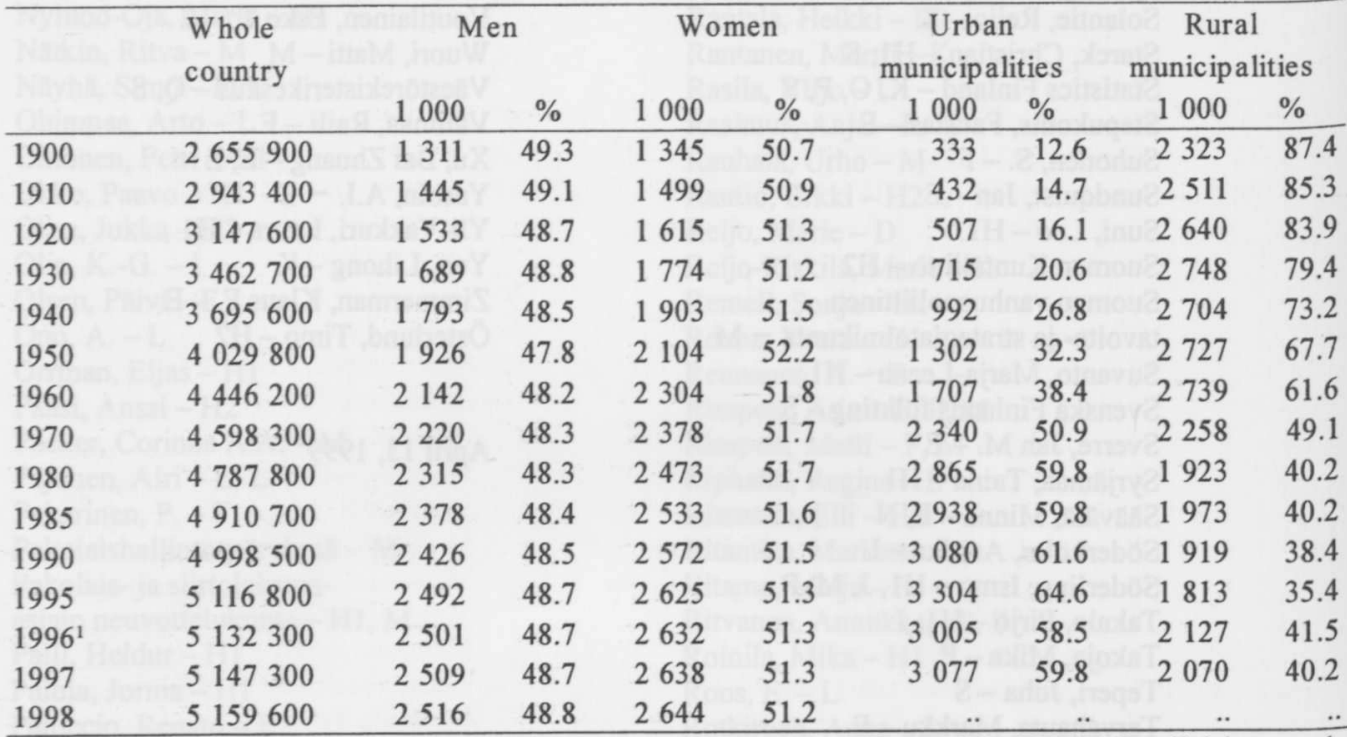

${ }^{1}$ The classification of municipalities into urban and rural areas changed in 1996. The division used here is the same as the one used by Statistics Finland.

Age structure

\begin{tabular}{rcccccccccr}
\hline & \multicolumn{3}{c}{ Whole population } & \multicolumn{3}{c}{ Men } & \multicolumn{3}{c}{ Women } \\
& $0-14$ & $15-64$ & $65-$ & $0-14$ & $15-64$ & $65-$ & $0-14$ & $15-64$ & $65-$ \\
& $\%$ & $\%$ & $\%$ & $\%$ & $\%$ & $\%$ & $\%$ & $\%$ & $\%$ \\
\hline 1900 & 35.0 & 59.6 & 5.4 & 35.9 & 59.4 & 4.7 & 34.3 & 59.7 & 6.0 \\
1910 & 35.6 & 58.6 & 5.8 & 36.7 & 58.3 & 5.0 & 34.6 & 59.0 & 6.4 \\
1920 & 33.4 & 60.7 & 5.9 & 34.8 & 60.0 & 5.2 & 32.0 & 61.3 & 6.7 \\
1930 & 29.4 & 64.3 & 6.3 & 30.6 & 64.1 & 5.3 & 28.3 & 64.5 & 7.2 \\
1940 & 26.9 & 66.7 & 6.4 & 28.2 & 66.5 & 5.3 & 25.7 & 66.9 & 7.4 \\
1950 & 30.0 & 63.3 & 6.7 & 32.0 & 62.8 & 5.2 & 28.1 & 63.9 & 8.0 \\
1960 & 30.1 & 62.4 & 7.4 & 31.9 & 62.3 & 5.7 & 28.5 & 62.5 & 8.9 \\
1970 & 24.3 & 66.3 & 9.3 & 25.7 & 67.1 & 7.2 & 23.0 & 65.7 & 11.3 \\
1980 & 20.2 & 67.8 & 12.1 & 21.4 & 69.7 & 8.9 & 19.1 & 66.0 & 14.9 \\
1985 & 19.4 & 68.0 & 12.6 & 20.5 & 70.4 & 9.1 & 18.4 & 65.8 & 15.8 \\
1990 & 19.3 & 67.2 & 13.5 & 20.3 & 69.8 & 9.9 & 18.3 & 64.8 & 16.9 \\
1995 & 19.0 & 66.7 & 14.3 & 19.9 & 69.2 & 10.9 & 18.1 & 64.3 & 17.4 \\
1996 & 18.9 & 66.6 & 14.5 & 19.8 & 69.1 & 11.1 & 18.0 & 64.3 & 17.7 \\
1997 & 18.7 & 66.7 & 14.6 & 19.6 & 69.1 & 11.3 & 17.8 & 64.4 & 17.8 \\
\hline
\end{tabular}

1 The data are based on the statistics of Statistics Finland, Helsinki.

* Preliminary data. 
Age-s pecific fertility rate

\begin{tabular}{lrrrrrrrr}
\hline \multicolumn{3}{c}{ Per 1 000 women } & & & & \multicolumn{2}{c}{ Total } \\
fertility \\
& $15-19$ & $20-24$ & $25-29$ & $30-34$ & $35-39$ & $40-44$ & $45-49$ & per woman \\
\hline $1901-10$ & 16.8 & 147.7 & 226.8 & 227.4 & 192.6 & 109.1 & 16.2 & 4.68 \\
$1911-20$ & 14.9 & 122.8 & 181.4 & 181.9 & 155.2 & 92.9 & 14.0 & 3.81 \\
$1921-30$ & 14.2 & 110.0 & 156.9 & 143.8 & 116.8 & 67.4 & 10.5 & 3.10 \\
$1931-40$ & 14.4 & 96.5 & 126.2 & 108.9 & 82.8 & 43.5 & 5.6 & 2.39 \\
$1941-45$ & 11.3 & 105.2 & 144.9 & 124.3 & 88.4 & 41.8 & 5.4 & 2.60 \\
$1946-50$ & 25.7 & 161.9 & 189.4 & 147.5 & 100.5 & 43.3 & 5.0 & 3.37 \\
$1951-55$ & 27.2 & 157.6 & 165.8 & 125.0 & 81.0 & 35.1 & 3.9 & 2.98 \\
$1956-60$ & 29.3 & 161.4 & 159.6 & 108.1 & 67.5 & 27.5 & 2.8 & 2.78 \\
$1961-65$ & 30.7 & 156.7 & 156.0 & 98.8 & 55.7 & 22.5 & 2.2 & 2.61 \\
$1966-70$ & 34.7 & 131.9 & 125.9 & 76.5 & 39.7 & 13.7 & 1.4 & 2.12 \\
$1971-75$ & 27.9 & 104.3 & 106.6 & 55.8 & 23.3 & 6.1 & 0.5 & 1.62 \\
$1976-80$ & 22.0 & 96.8 & 114.6 & 67.5 & 26.4 & 6.0 & 0.3 & 1.67 \\
$1981-85$ & 15.7 & 85.0 & 122.8 & 75.3 & 32.1 & 6.7 & 0.4 & 1.69 \\
$1986-90$ & 12.3 & 70.2 & 126.0 & 85.6 & 33.2 & 7.5 & 0.4 & 1.68 \\
1991 & 12.2 & 72.4 & 133.3 & 96.7 & 37.0 & 7.3 & 0.4 & 1.79 \\
1992 & 11.7 & 73.5 & 137.6 & 100.2 & 38.6 & 7.7 & 0.5 & 1.85 \\
1993 & 10.5 & 71.8 & 134.4 & 100.5 & 38.2 & 7.5 & 0.4 & 1.81 \\
1994 & 10.1 & 71.2 & 135.3 & 104.5 & 40.6 & 7.7 & 0.4 & 1.85 \\
1995 & 9.8 & 66.3 & 130.2 & 105.0 & 41.5 & 8.3 & 0.4 & 1.81 \\
1996 & 9.8 & 63.7 & 125.3 & 102.1 & 42.9 & 8.1 & 0.4 & 1.76 \\
1997 & 9.0 & 62.2 & 123.4 & 100.8 & 44.0 & 8.5 & 0.5 & 1.75 \\
\hline & & & & & & & &
\end{tabular}

Gross and net reproduction rate

\begin{tabular}{lccccc}
\hline & $\begin{array}{c}\text { Gross } \\
\text { reproduction } \\
\text { rate }\end{array}$ & $\begin{array}{c}\text { Net } \\
\text { reproduction } \\
\text { rate }\end{array}$ & & $\begin{array}{c}\text { Gross } \\
\text { reproduction } \\
\text { rate }\end{array}$ & $\begin{array}{c}\text { Net } \\
\text { reproduction } \\
\text { rate }\end{array}$ \\
\hline $1901-10$ & 2.278 &.. & $1961-65$ & 1.276 & 1.236 \\
$1911-20$ & 1.849 &.. & $1966-70$ & 1.035 & 1.009 \\
$1921-30$ & 1.504 &.. & $1971-75$ & 0.790 & 0.773 \\
$1931-35$ & 1.167 & 0.958 & $1976-80$ & 0.814 & 0.801 \\
$1936-40$ & 1.162 & 0.962 & $1981-85$ & 0.826 & 0.815 \\
$1941-45$ & 1.257 & 1.053 & $1986-90$ & 0.819 & 0.808 \\
$1946-50$ & 1.622 & 1.382 & $1991-95$ & 0.894 & 0.882 \\
$1951-55$ & 1.452 & 1.373 & 1996 & 0.859 & 0.849 \\
$1956-60$ & 1.357 & 1.301 & 1997 & 0.859 & 0.848 \\
\hline
\end{tabular}

Median age of mother at first birth

\begin{tabular}{lccc}
\hline & Median age & Median age \\
\hline 1950 & 24.20 & 1991 & 26.78 \\
$1961-65$ & 23.25 & 1992 & 26.88 \\
$1966-70$ & 22.73 & 1993 & 27.12 \\
$1971-75$ & 24.03 & 1994 & 27.20 \\
$1976-80$ & 25.18 & 1995 & 27.42 \\
$1981-85$ & 25.40 & 1996 & 27.48 \\
$1986-90$ & 26.30 & 1997 & 27.63 \\
\hline
\end{tabular}


Live births

\begin{tabular}{lccccc}
\hline & $\begin{array}{c}\text { Annual } \\
\text { mean }\end{array}$ & $\begin{array}{c}\text { Per 1 000 of } \\
\text { mean population }\end{array}$ & & Number & $\begin{array}{c}\text { Per 1 000 of } \\
\text { mean population }\end{array}$ \\
\hline $1901-10$ & 90292 & 32.4 & 1991 & 65395 & 13.0 \\
$1911-20$ & 83045 & 27.0 & 1992 & 66731 & 13.2 \\
$1921-30$ & 78184 & 23.6 & 1993 & 64826 & 12.8 \\
$1931-40$ & 70584 & 19.7 & 1994 & 65231 & 12.8 \\
$1941-50$ & 92613 & 24.3 & 1995 & 63067 & 12.3 \\
$1951-60$ & 88024 & 20.7 & 1996 & 60723 & 11.8 \\
$1961-70$ & 76466 & 16.8 & 1997 & 59329 & 11.5 \\
$1971-80$ & 62789 & 13.3 & $1998^{*}$ & 57212 & 11.1 \\
$1981-85$ & 64868 & 13.4 & & & \\
$1986-90$ & 62534 & 12.6 & & & \\
\hline
\end{tabular}

Illegitimate live births

\begin{tabular}{|c|c|c|c|c|c|}
\hline F & & $\begin{array}{c}\text { Percent of } \\
\text { all live births }\end{array}$ & & Number & $\begin{array}{l}\text { Percent of } \\
\text { all live births }\end{array}$ \\
\hline $1901-10$ & 6120 & 6.8 & 1991 & 17896 & 27.4 \\
\hline $1911-20$ & 6573 & 6.8 & 1992 & 19257 & 28.9 \\
\hline $1921-30$ & 6606 & 8.4 & 1993 & 19665 & 30.3 \\
\hline $1931-40$ & 5349 & 7.6 & 1994 & 20438 & 31.3 \\
\hline $1941-50$ & 5629 & 6.1 & 1995 & 20886 & 33.1 \\
\hline $1951-60$ & 3819 & 4.3 & 1996 & 21484 & 35.4 \\
\hline $1961-70$ & 3621 & 4.7 & 1997 & 21659 & 36.5 \\
\hline $1971-80$ & 6182 & 9.8 & & 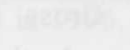 & 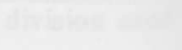 \\
\hline $1981-85$ & 9388 & 14.5 & & 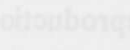 & \\
\hline $1986-90$ & 13301 & 21.3 & & & \\
\hline
\end{tabular}

Abortions

\begin{tabular}{lccccc}
\hline & Number & $\begin{array}{c}\text { Percent of } \\
\text { live births }\end{array}$ & & Number & $\begin{array}{c}\text { Percent of } \\
\text { live births }\end{array}$ \\
\hline 1970 & 14757 & 22.9 & 1985 & 13833 & 22.0 \\
1971 & 20622 & 33.8 & 1986 & 13319 & 22.0 \\
1972 & 22146 & 37.5 & 1987 & 12995 & 21.7 \\
1973 & 23362 & 41.1 & 1988 & 12749 & 20.1 \\
1974 & 22846 & 36.5 & 1989 & 12658 & 20.0 \\
1975 & 21547 & 32.8 & 1990 & 12232 & 18.7 \\
1976 & 19818 & 29.6 & 1991 & 11747 & 18.0 \\
1977 & 17772 & 27.1 & 1992 & 11071 & 16.6 \\
1978 & 16928 & 26.4 & 1993 & 10342 & 15.9 \\
1979 & 15849 & 25.1 & 1994 & 10013 & 15.4 \\
1980 & 15037 & 23.8 & 1995 & 9884 & 15.7 \\
1981 & 14120 & 22.2 & 1996 & 10437 & 17.2 \\
1982 & 13861 & 21.0 & 1997 & 10238 & 17.3 \\
1983 & 13360 & 20.0 & $1998^{*}$ & 10600 & 18.5 \\
1984 & 13645 & 21.0 & & & \\
\hline
\end{tabular}


Marriages

\begin{tabular}{lccccc}
\hline & $\begin{array}{c}\text { Annual } \\
\text { mean }\end{array}$ & $\begin{array}{c}\text { Per 1 000 of } \\
\text { mean population }\end{array}$ & & Number & $\begin{array}{c}\text { Per 1 000 of } \\
\text { mean population }\end{array}$ \\
\hline $1901-10$ & 18947 & 6.8 & 1991 & 24732 & 4.9 \\
$1911-20$ & 18922 & 6.2 & 1992 & 23560 & 4.7 \\
$1921-30$ & 23725 & 7.2 & 1993 & 24660 & 4.9 \\
$1931-40$ & 28491 & 7.9 & 1994 & 24898 & 4.9 \\
$1941-50$ & 37367 & 9.5 & 1995 & 23737 & 4.6 \\
$1951-60$ & 32191 & 7.6 & 1996 & 24464 & 4.8 \\
$1961-70$ & 37398 & 8.2 & 1997 & 23444 & 4.6 \\
$1971-80$ & 32575 & 6.9 & $1998^{*}$ & 23035 & 4.5 \\
$1981-85$ & 24588 & 5.1 & & & \\
$1986-90$ & 25516 & 5.2 & & & \\
\hline
\end{tabular}

Median age at first marriage

\begin{tabular}{lccccc}
\hline & Men & Women & & Men & Women \\
\hline $1901-05$ & 26.1 & 23.5 & $1956-60$ & 24.6 & 22.8 \\
$1906-10$ & 26.2 & 23.6 & $1961-65$ & 24.1 & 22.3 \\
$1911-15$ & 26.4 & 23.7 & $1966-70$ & 23.6 & 22.2 \\
$1916-20$ & 27.0 & 24.0 & $1971-75$ & 24.0 & 22.7 \\
$1921-25$ & 26.9 & 24.0 & $1976-80$ & 25.2 & 23.3 \\
$1926-30$ & 26.6 & 23.9 & $1981-85$ & 26.2 & 24.2 \\
$1931-35$ & 27.1 & 24.4 & $1986-90$ & 27.1 & 25.2 \\
$1936-40$ & 27.6 & 24.7 & $1991-95$ & 28.1 & 26.3 \\
$1941-45$ & 27.6 & 24.3 & 1996 & 28.9 & 27.0 \\
$1946-50$ & 26.1 & 23.7 & 1997 & 29.0 & 27.2 \\
$1951-55$ & 24.9 & 23.2 & & & \\
\hline
\end{tabular}

Consensual unions

\begin{tabular}{|c|c|c|c|c|c|c|c|}
\hline & Pro & rtion $(\%$ & of consens & al union & of all uni & & Persons living in \\
\hline & $15-24$ & $25-29$ & $30-34$ & $35-44$ & $45-64$ & All & consensual union \\
\hline 1981 & 44.6 & 19.4 & 12.4 & 6.5 & 2.5 & 10.3 & 214100 \\
\hline 1985 & 55.0 & 27.1 & 13.5 & 7.4 & 4.4 & 12.3 & 283000 \\
\hline 1987 & 62.5 & 32.9 & 15.2 & 9.6 & 4.7 & 14.8 & 324000 \\
\hline 1990 & 69.8 & 39.3 & 21.6 & 12.4 & 6.8 & 15.9 & 380500 \\
\hline 1992 & 73.4 & 43.7 & 25.2 & 14.4 & 7.3 & 17.1 & 409300 \\
\hline 1994 & 75.5 & 45.7 & 27.5 & 16.3 & 8.3 & 17.9 & 427300 \\
\hline 1996 & 78.8 & 48.6 & 30.1 & 18.3 & 9.3 & 19.1 & 458200 \\
\hline 1997 & 80.0 & 50.1 & 31.6 & 19.5 & 9.8 & 19.8 & 475230 \\
\hline Divorce & & & & & & & \\
\hline & & & $\begin{array}{r}\text { Per } 100 \\
\text { mean pop }\end{array}$ & $\begin{array}{l}0 \text { of } \\
\text { lation }\end{array}$ & & Number & $\begin{array}{l}\text { Per } 1000 \text { of } \\
\text { mean population }\end{array}$ \\
\hline $1951-60$ & & 584 & 0.84 & & 1991 & 12820 & 2.56 \\
\hline $1961-70$ & & 860 & 1.06 & & 1992 & 12949 & 2.57 \\
\hline $1971-75$ & & 727 & 1.87 & & 1993 & 12753 & 2.52 \\
\hline $1976-80$ & & 051 & 2.12 & & 1994 & 13751 & 2.70 \\
\hline $1981-85$ & & 534 & 1.96 & & 1995 & 14025 & 2.75 \\
\hline $1986-90$ & & & 2.40 & & 1996 & 13795 & 2.69 \\
\hline & & & & & 1997 & 13507 & 2.63 \\
\hline & & & & & $1998^{*}$ & 13811 & 2.68 \\
\hline
\end{tabular}


Families with children under 18 years of age by type of family and number of children (\%)

\begin{tabular}{|c|c|c|c|c|c|}
\hline & \multicolumn{4}{|c|}{ Number of children } & \multirow[t]{2}{*}{ Number } \\
\hline & 1 & 2 & 3 & $4+$ & \\
\hline \multicolumn{6}{|c|}{ Married couples with children ${ }^{1}$} \\
\hline 1960 & 34.4 & 30.7 & 17.5 & 17.4 & 601542 \\
\hline 1970 & 40.2 & 33.9 & 15.5 & 10.4 & 602076 \\
\hline 1980 & 44.6 & 41.1 & 11.1 & 3.2 & 588373 \\
\hline 1990 & 40.3 & 41.6 & 14.1 & 4.0 & 534665 \\
\hline $1990^{2}$ & 38.9 & 42.4 & 14.5 & 4.1 & 490965 \\
\hline 1995 & 38.4 & 40.7 & 15.7 & 5.1 & 449241 \\
\hline 1996 & 38.1 & 40.6 & 15.9 & 5.3 & 438883 \\
\hline 1997 & 37.8 & 40.6 & 16.1 & 5.5 & 428922 \\
\hline \multicolumn{6}{|c|}{ Couples living in consensual union with children } \\
\hline $1990^{2}$ & 60.0 & 30.2 & 7.9 & 1.9 & 59827 \\
\hline 1995 & 54.7 & 34.1 & 8.9 & 2.3 & 78528 \\
\hline 1996 & 54.0 & 34.4 & 9.3 & 2.3 & 82135 \\
\hline 1997 & 53.2 & 35.0 & 9.4 & 2.4 & 86176 \\
\hline \multicolumn{6}{|c|}{ Mothers with children } \\
\hline 1960 & 61.1 & 23.0 & 9.2 & 6.7 & 67381 \\
\hline 1970 & 60.7 & 23.8 & 9.5 & 6.0 & 66303 \\
\hline 1980 & 67.7 & 25.3 & 5.6 & 1.4 & 88896 \\
\hline 1990 & 66.8 & 26.5 & 5.5 & 1.2 & 93669 \\
\hline $1990^{2}$ & 65.6 & 27.2 & 5.9 & 1.3 & 78869 \\
\hline 1995 & 60.8 & 29.7 & 7.7 & 1.9 & 98351 \\
\hline 1996 & 59.9 & 30.1 & 8.0 & 2.0 & 100219 \\
\hline 1997 & 59.2 & 30.5 & 8.2 & 2.1 & 101309 \\
\hline \multicolumn{6}{|c|}{ Fathers with children } \\
\hline 1960 & 56.7 & 24.8 & 10.3 & 8.2 & 9123 \\
\hline 1970 & 61.1 & 24.3 & 9.0 & 5.6 & 8656 \\
\hline 1980 & 71.1 & 23.0 & 4.8 & 1.1 & 11463 \\
\hline 1990 & 72.5 & 22.9 & 3.8 & 0.7 & 13240 \\
\hline $1990^{2}$ & 71.7 & 23.4 & 4.1 & 0.8 & 11074 \\
\hline 1995 & 71.0 & 23.4 & 4.5 & 1.1 & 13490 \\
\hline 1996 & 70.4 & 23.7 & 4.8 & 1.1 & 13797 \\
\hline 1997 & 70.0 & 24.1 & 5.0 & 0.9 & 14065 \\
\hline
\end{tabular}

${ }^{1}$ Includes not-married couples with children under 18 years, until 1990.

2 The number of consensual unions has been calculated in a new way.

Families with children under 18 years of age by type of family (\%)

\begin{tabular}{lccccc}
\hline & $\begin{array}{c}\text { Married couples } \\
\text { with } \\
\text { children }^{1}\end{array}$ & $\begin{array}{c}\text { Couples living in } \\
\text { consensual union } \\
\text { with children }\end{array}$ & $\begin{array}{c}\text { Mothers } \\
\text { with } \\
\text { children }\end{array}$ & $\begin{array}{c}\text { Fathers } \\
\text { with } \\
\text { children }\end{array}$ & Number \\
\hline 1960 & 88.7 &.. & 10.0 & 1.3 & 678046 \\
1970 & 88.9 &.. & 9.8 & 1.3 & 677035 \\
1980 & 85.4 &.. & 12.9 & 1.7 & 688732 \\
1990 & 83.3 &.. & 14.6 & 2.1 & 641574 \\
$1990^{2}$ & 76.6 & 9.3 & 12.3 & 1.7 & 640735 \\
1995 & 70.2 & 12.3 & 15.4 & 2.1 & 639610 \\
1996 & 69.1 & 12.9 & 15.8 & 2.2 & 635034 \\
1997 & 68.0 & 13.7 & 16.1 & 2.2 & 630422 \\
\hline
\end{tabular}

${ }^{1}$ Includes not-married couples with children under 18 years, until 1990.

2 The number of consensual unions has been calculated in a new way. 
Deaths

\begin{tabular}{|c|c|c|c|c|c|c|c|}
\hline & $\begin{array}{c}\text { Annual } \\
\text { mean }\end{array}$ & $\begin{array}{c}\text { Per } 1000 \\
\text { of mean } \\
\text { population }\end{array}$ & $\begin{array}{c}\text { Infant } \\
\text { mortality }^{1}\end{array}$ & & Number & $\begin{array}{c}\text { Per } 1000 \\
\text { of mean } \\
\text { population }\end{array}$ & $\begin{array}{c}\text { Infant } \\
\text { mortality }^{1}\end{array}$ \\
\hline $1901-10$ & 52099 & 18.7 & 123.8 & 1991 & 49294 & 9.8 & 5.8 \\
\hline $1911-20$ & 58284 & 19.0 & 111.8 & 1992 & 49844 & 9.9 & 5.2 \\
\hline $1921-30$ & 49573 & 14.9 & 91.9 & 1993 & 50988 & 10.1 & 4.4 \\
\hline $1931-40$ & 50222 & 14.0 & 72.0 & 1994 & 48000 & 9.4 & 4.7 \\
\hline $1941-50$ & 51838 & 13.6 & 56.0 & 1995 & 49280 & 9.6 & 3.9 \\
\hline $1951-60$ & 39381 & 9.3 & 28.6 & 1996 & 49167 & 9.6 & 4.0 \\
\hline $1961-70$ & 43494 & 9.5 & 16.7 & 1997 & 49108 & 9.6 & 3.9 \\
\hline $1971-80$ & 44243 & 9.4 & 11.3 & $1998^{*}$ & 49389 & 9.6 & .. \\
\hline $1981-85$ & 45299 & 9.3 & 6.3 & $y^{2}$ & & - & \\
\hline $1986-90$ & 48663 & 9.8 & 5.9 & 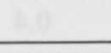 & & & \\
\hline
\end{tabular}

${ }^{1}$ Mortality during the first year of life per 1000 live births.

Mean expectation of life at birth

\begin{tabular}{lccccc}
\hline & Men & Women & & Men & Women \\
\hline $1911-20^{1}$ & 43.4 & 49.1 & $1976-80$ & 68.5 & 77.2 \\
$1921-30$ & 50.7 & 55.1 & $1981-85$ & 70.1 & 78.4 \\
$1931-40^{2}$ & 54.5 & 59.6 & $1986-90$ & 70.7 & 78.8 \\
$1941-45^{2}$ & 54.6 & 61.1 & 1991 & 71.3 & 79.3 \\
$1946-50$ & 58.6 & 65.8 & 1992 & 71.7 & 79.4 \\
$1951-55$ & 63.4 & 69.8 & 1993 & 72.1 & 79.5 \\
$1956-60$ & 65.9 & 71.6 & 1994 & 72.8 & 80.2 \\
$1961-65$ & 65.4 & 72.6 & 1995 & 72.8 & 80.2 \\
$1966-70$ & 65.9 & 73.6 & 1996 & 73.0 & 80.5 \\
$1971-75$ & 66.7 & 75.2 & 1997 & 73.4 & 80.5 \\
\hline
\end{tabular}

${ }^{1}$ Including deaths due to war operations.

${ }^{2}$ Excluding deaths due to war operations.

Excess of births over deaths

\begin{tabular}{lccccc}
\hline & Annual mean & $\begin{array}{c}\text { Per 1 000 of } \\
\text { mean population }\end{array}$ & & Number & $\begin{array}{c}\text { Per 1 000 of } \\
\text { mean population }\end{array}$ \\
\hline $1901-10$ & 38194 & 13.7 & 1991 & 16101 & 3.2 \\
$1911-20$ & 24760 & 8.1 & 1992 & 16887 & 3.3 \\
$1921-30$ & 28611 & 8.7 & 1993 & 13838 & 2.7 \\
$1931-40$ & 20354 & 5.7 & 1994 & 17231 & 3.4 \\
$1941-50$ & 44272 & 10.7 & 1995 & 13787 & 2.7 \\
$1951-60$ & 48643 & 11.4 & 1996 & 11556 & 2.3 \\
$1961-70$ & 32223 & 7.2 & 1997 & 10221 & 2.0 \\
$1971-75$ & 16632 & 3.6 & $1998^{*}$ & 7823 & 1.5 \\
$1976-80$ & 20460 & 4.3 & & & \\
$1981-85$ & 19569 & 4.0 & & & \\
$1986-90$ & 13871 & 2.8 & & & \\
\hline
\end{tabular}


International migration

\begin{tabular}{lcccrc}
\hline & $\begin{array}{c}\text { Emigrants who } \\
\text { have obtained } \\
\text { a passport }\end{array}$ & $\begin{array}{c}\text { Per 1 000 of } \\
\text { mean population }\end{array}$ & & $\begin{array}{c}\text { Yearly net } \\
\text { migration }\end{array}$ & $\begin{array}{c}\text { Per 1 000 of } \\
\text { mean population }\end{array}$ \\
\hline $1901-10$ & 150832 & 5.4 & $1976-80$ & -7493 & -1.6 \\
$1911-20$ & 67346 & 2.2 & $1981-85$ & +5347 & 1.1 \\
$1921-30$ & 58559 & 1.8 & $1986-90$ & +2905 & 0.6 \\
$1931-40$ & 8844 & 0.2 & 1991 & +13017 & 2.6 \\
& & & 1992 & +8499 & 1.7 \\
$1941-50$ & Yearly net migration & & 1993 & +8390 & 1.7 \\
$1951-60$ & -4100 & -1.1 & 1994 & +2939 & 0.6 \\
$1961-70$ & -7003 & -1.5 & 1995 & +3265 & 0.6 \\
$1971-75$ & -17763 & -3.9 & 1996 & +2707 & 0.5 \\
& +1936 & 0.4 & 1997 & +3710 & 0.7 \\
& & & $1998^{*}$ & +3417 & 0.7 \\
\hline
\end{tabular}

Internal migration

\begin{tabular}{lccccc}
\hline & $\begin{array}{c}\text { Annual } \\
\text { mean }^{1}\end{array}$ & $\begin{array}{c}\text { Percent of } \\
\text { mean population }\end{array}$ & & Number & $\begin{array}{c}\text { Percent of } \\
\text { mean population }\end{array}$ \\
\hline $1901-10$ & 74784 & 2.7 & 1991 & 183479 & 3.7 \\
$1911-20$ & 85111 & 2.8 & 1992 & 169270 & 3.4 \\
$1921-30$ & 79248 & 2.4 & 1993 & 181333 & 3.6 \\
$1931-39$ & 136470 & 3.8 & 1994 & 217776 & 4.3 \\
$1941-50$ & 132778 & 3.5 & 1995 & 215971 & 4.2 \\
$1951-60$ & 172098 & 4.0 & 1996 & 236460 & 4.6 \\
$1961-70$ & 219330 & 4.8 & 1997 & 239115 & 4.7 \\
$1971-80$ & 213569 & 4.6 & $1998^{*}$ & 256370 & 5.0 \\
$1981-85$ & 193681 & 4.0 & & & \\
$1986-90$ & 200737 & 4.1 & & & \\
\hline
\end{tabular}

${ }^{1}$ Average in-migration to rural and urban municipalities

Number of aliens and their proportion of total population and groups of aliens by citizens hip in 1997

\begin{tabular}{llllr}
\hline & Number of aliens & $\%$ & \multicolumn{2}{c}{$\begin{array}{c}\text { Groups of aliens } \\
\text { by citizenship in 1997 }\end{array}$} \\
\hline 1976 & 12154 & 0.3 & Europe & 51974 \\
1980 & 12502 & 0.3 & EU-countries & 14719 \\
1985 & 16478 & 0.3 & (of which Sweden) & 7507 \\
1986 & 16585 & 0.3 & Russia & 14316 \\
1987 & 17730 & 0.4 & Estonia & 9689 \\
1988 & 18676 & 0.4 & Former USSR & 4675 \\
1989 & 21174 & 0.4 & Yugoslavia & 2541 \\
1990 & 26255 & 0.5 & Other & 6034 \\
1991 & 37579 & 0.7 & Africa & 8547 \\
1992 & 46250 & 0.9 & Somalia & 5238 \\
1993 & 55587 & 1.1 & Other & 3309 \\
1994 & 62012 & 1.2 & America & 3335 \\
1995 & 68566 & 1.3 & Asia and Oceania & 14568 \\
1996 & 73754 & 1.4 & Unknown & 2176 \\
1997 & 80600 & 1.6 & & \\
\hline
\end{tabular}


Economically active population by industry (\%)

\begin{tabular}{lrrrrrr}
\hline & 1950 & 1960 & 1970 & 1980 & $1990^{1}$ & $1995^{1}$ \\
\hline Agriculture and forestry, & & & & & & \\
hunting and fishing & 45.8 & 35.5 & 20.3 & 12.6 & 8.4 & 7.6 \\
Industry etc. & 20.8 & 21.6 & 25.9 & 26.3 & 22.5 & 22.1 \\
Construction & 6.3 & 8.7 & 8.3 & 7.1 & 8.3 & 5.6 \\
Commerce & 9.5 & 13.6 & 18.9 & 19.1 & 25.6 & 25.1 \\
Transport and communications & 5.4 & 6.3 & 7.1 & 7.9 & 7.2 & 7.9 \\
Services & 10.8 & 14.0 & 18.1 & 24.8 & 27.8 & 31.4 \\
Unknown & 1.4 & 0.3 & 1.4 & 2.2 & 0.1 & 0.3 \\
& 100.0 & 100.0 & 100.0 & 100.0 & 100.0 & 100.0 \\
\hline
\end{tabular}

${ }^{1}$ Employed labor force. The grounds for classification have been changed in some extent in 1989 and in 1995 .

Females in labor force'

\begin{tabular}{lccccc}
\hline & $\begin{array}{c}\text { The proportion of } \\
\text { females in labor force } \\
\text { (percent) }\end{array}$ & & \multicolumn{2}{c}{$\begin{array}{c}\text { Female labor force as a percentage } \\
\text { of female population aged }\end{array}$} \\
\hline $1960-64$ & 44.0 & $1960-64$ & $20-44$ years & $15-64$ years & $15-74$ years \\
$1965-69$ & 43.3 & $1965-69$ &.. &.. & 56.2 \\
$1970-74$ & 44.7 & $1970-74$ &.. &.. & 55.1 \\
$1975-79$ & 46.3 & $1975-79$ &. &. & 57.9 \\
$1980-84$ & 47.3 & $1980-84$ & 78.0 & 66.8 & 59.9 \\
$1985-89$ & 47.7 & $1985-89$ & 82.5 & 71.2 & 63.0 \\
1990 & 47.5 & 1990 & 84.2 & 72.8 & 64.4 \\
1991 & 47.5 & 1991 & 82.8 & 72.5 & 63.9 \\
1992 & 47.4 & 1992 & 81.7 & 71.7 & 63.1 \\
1993 & 47.4 & 1993 & 80.0 & 70.4 & 61.8 \\
1994 & 47.5 & 1994 & 79.5 & 69.8 & 61.2 \\
1995 & 47.5 & 1995 & 79.2 & 69.6 & 60.9 \\
1996 & 47.6 & 1996 & 78.8 & 70.0 & 61.2 \\
1997 & 47.3 & 1997 & 79.1 & 70.3 & 61.4 \\
\hline
\end{tabular}

${ }^{1}$ The data for 1981-87 have been corrected to correspond to the data of the revised Labour Force Survey from 1987 on.

Unemployment rate

\begin{tabular}{lccc}
\hline & Both sexes & Men & Women \\
\hline $1965-69$ & 2.5 & 3.4 & 1.3 \\
$1970-74$ & 2.3 & 2.6 & 1.8 \\
$1975-79$ & 5.1 & 5.8 & 4.3 \\
$1980-84$ & 5.1 & 5.3 & 4.9 \\
$1985-89$ & 4.7 & 5.2 & 4.2 \\
1990 & 3.4 & 4.0 & 2.8 \\
1991 & 7.6 & 9.3 & 5.7 \\
1992 & 13.1 & 15.5 & 10.5 \\
1993 & 17.9 & 19.8 & 15.7 \\
1994 & 18.4 & 19.9 & 16.7 \\
1995 & 17.2 & 17.6 & 16.7 \\
1996 & 16.3 & 16.1 & 16.5 \\
19971 & 14.5 & 14.0 & 15.0 \\
1998 & 11.4 & 10.9 & 12.0 \\
\hline
\end{tabular}

${ }^{1}$ The grouds for classification of unemployment have been changed somewhat in 1997 . 